\title{
Dynamics and lifetime of persistent activity states in random networks of spiking neurons with strong synapses
}

\author{
Birgit Kriener ${ }^{1 *}$, Håkon Enger ${ }^{1}$, Tom Tetzlaff ${ }^{2}$, Hans Ekkehard Plesser ${ }^{1}$, Marc-Oliver Gewaltig ${ }^{3}$, Gaute T Einevoll \\ From Twenty Second Annual Computational Neuroscience Meeting: CNS*2013 \\ Paris, France. 13-18 July 2013
}

The selective persistent activation of populations of neurons in the prefrontal cortex during active memory tasks is one of the best-studied neural correlates of a higher cognitive function, the so-called working memory. Yet, the respective roles of network and neuron properties in this activity are not fully understood. Spiking neuron network models often rely on a selective excitatory connectivity and generate spike trains that are more regular than what is experimentally observed, thus necessitating additional assumptions, such as intrinsic cellular bistability or additional external noisy input.

Recently, it was shown that very simple random networks of excitatory and inhibitory spiking neurons can generate persistent asynchronous-irregular activity only by admitting a certain fraction of comparably strong synaptic weights [1,2]. A large number of experiments showed that synaptic weight distributions are indeed commonly characterized by a large fraction of weak weights and a heavy tail of quite strong weights [e.g. 2,3]. Here, we demonstrate how including such strong synaptic couplings leads to the occurrence of a firing-rate attractor at moderate rates with highly irregular individual spike trains.

Based on the firing-rate transfer of the network, we show that the firing-rate dynamics becomes bistable if the synapses are sufficiently strong: in addition to the quiescent state, a second stable fixed point at moderate firing rates emerges by a saddle-node bifurcation. However, the population firing-rate is characterized by pronounced inherent fluctuations that perpetually perturb

\footnotetext{
* Correspondence: birgit.kriener@umb.no

'Department of Mathematical Sciences and Technology, Norwegian

University of Life Science, Ås, Norway

Full list of author information is available at the end of the article
}

this fixed point. It is the trade-off between the size of the population fluctuations and the size of the basin of attraction that thus determines the onset and lifetime of persistent activity states. Moreover, individual neuronal activity turns out to be very irregular, switching between long periods of low firing rate to short burst-like states. We show that this is an effect of the strong coupling strength in the network combined with the finite memory time constant of the neurons. Thus, such irregular neuron dynamics can be a pure network phenomenon, and do not require intracellular bistability or additional high-variability noise as previously suggested.

\section{Acknowledgements \\ We gratefully acknowledge funding by the escience program of the Research Council of Norway under grant 178892/N30 (eNeuro), the Helmholtz Association: HASB and portfolio theme SMHB, the Next- Generation Supercomputer Project of MEXT, and EU Grant 269921 (BrainScaleS). All network simulations were carried out with NEST (http:// www.nest-initiative.org) using NOTUR computing resources.}

\section{Author details}

'Department of Mathematical Sciences and Technology, Norwegian University of Life Science, Ås, Norway. ${ }^{2}$ Inst. of Neuroscience and Medicine (INM-6) and Inst. for Advanced Simulation (IAS-6), Jülich Research Centre and JARA, Jülich, Germany. ${ }^{3}$ Blue Brain Project, École Polytechnique Fédérale de Lausanne, Lausanne, Switzerland.

Published: 8 July 2013

\section{References}

1. Gewaltig MO: Self-sustained activity, burst, and variability in recurrent., Under review.

2. Teramae JN, Tsubo Y, Fukai T: Optimal spike-based communication in excitable networks with strong-sparse and weak-dense links. Sci Rep 2012, 2:485.

3. Lefort S, Tomm C, Sarria JCF, Petersen CCH: The excitatory neuronal network of the C2 barrel column in mouse primary somatosensory. Neuron 2007, 61:301-316.

\section{C)


4. Avermann M, Tomm C, Mateo C, Gerstner W, Petersen CCH: Microcircuits of excitatory and inhibitory neurons in layer $2 / 3$ of mouse barrel cortex. Journal of Neurophysiology 2012, 107(11):3116-3134.

doi:10.1186/1471-2202-14-S1-P121

Cite this article as: Kriener et al:: Dynamics and lifetime of persistent activity states in random networks of spiking neurons with strong synapses. BMC Neuroscience 2013 14(Suppl 1):P121.

Submit your next manuscript to BioMed Central and take full advantage of:

- Convenient online submission

- Thorough peer review

- No space constraints or color figure charges

- Immediate publication on acceptance

- Inclusion in PubMed, CAS, Scopus and Google Scholar

- Research which is freely available for redistribution

Submit your manuscript at www.biomedcentral.com/submit
C Biomed Central 